\title{
Numerical Simulation of Conjugate Heat Exchange at Gas Flowing in a Well
}

\author{
Michael Al'es ${ }^{1}$, Sergey Makarov ${ }^{1, *}$, and Alexandr Karpov ${ }^{1}$ \\ ${ }^{1}$ Udmurt Federal Research Center of the Ural Branch of the Russian Academy of Sciences, 34, \\ T. Baramzinoy St., Izhevsk, 426067, Russia
}

\begin{abstract}
A mathematical model is offered for conjugate heat exchange when gas flows in the section of a vertical well. The motion of the medium is described using a two-dimensional axisymmetric stationary formulation based on boundary-layer equations. The turbulent gas flowing due to the reservoir energy is considered. Natural gas is taken as a travelling medium. The numerical simulation results are presented in the form of the dependences of the flow temperature and gas density along the radius of the well on the external factors of the heat exchange changing along the well height. The results describe the thermobaric state of the well in the condition of the conjugate heat exchange of the produced natural gas flow and tubed well depending on the external thermal conditions.
\end{abstract}

\section{Introduction}

The reliability of the wells producing raw hydrocarbons is very important in the real production conditions. In comparison with an oil well, gas well characteristics are mainly determined by the parameters of the travelling gas, which depend on the external factors, particularly on temperature. Initially, in the producing layer gas is at the pressure and temperature of the layer; as the gas is travelling upwards in the well, the variables of state of the gas change. Thus, when gas travels upwards in the well, its pressure, temperature, density and, correspondingly, the flow velocity vary. In this connection there is the necessity to determine the variation of the above parameters with time and within space. This will permit to increase the reliability of the information on the well operation at varying external conditions. It is equally applicable both to the underground and to the underwater production of gas.

Thus, the determination of the intensity of the variation of the main parameters of the gas flow state in the well under the influence of external factors such as external temperature conditions is an important scientific and technological task.

It is known that the intensity of the variation of the gas flow parameters mainly depends on hydrodynamic and thermal parameters which change in the process of the production and transfer of gas along the well. In this connection current research works are mainly concentrated on finding the criteria for the evaluation of the influence of temperature and

\footnotetext{
*Corresponding author: ssmak15@mail.ru
} 
hydrodynamic regimes on the well operation conditions. Thus, in [1] the main equations and the results of the calculations of the temperature fields of the gas well depending on spatial coordinates and time are given for different regimes of the well operation; the upward gas movement along the well with constant velocity is considered. The model solutions are obtained using the analytical approach. The results of the solution of the stationary non-linear problem of the distribution of the gas density over the well shaft are presented in [2]. It is shown that the variation of the density for the typical modes of the gas flow in the well shaft significantly contribute into the distribution of the thermodynamic parameters of the production process. The mathematical simulation of the variation of the parameters of the gas flow state in the well due to the influence of the external factors was performed in [3]. The distribution of the parameters of pressure and temperature at the nonisometric filtration simultaneously with the operation of the gas well was obtained for the flat radial influx of gas. The modal problems of the gas flow in the well were solved in stationary [4] and non-stationary [5] formulation. The mathematical models are widely used for describing the turbulent gas flow, for example, in [6 - 8]. In $[9,10]$ the solutions for the problems of conjugate heat exchange are given for the laminar and turbulent flows of formation fluid in a well. It is shown that the movement of the formation fluid containing heavy hydrocarbons can lead to the formation of paraffin on the inner walls of wells. The dynamics of the paraffin formation is mainly determined by the intensity of the temperature variation in the conjugate system "well - layer".

In the present paper, a mathematical model is offered and the results of the numerical investigations are presented of the regularities of the temperature field distribution and the variations of the parameters of the produced gas due to the influence of the fields. For the formulation and solution of the problem of the gas flow in a well we offer to consider the use of a two-dimensional axisymmetric stationary formulation on the basis of the boundarylayer equations [6].

The objective of the present work is the construction of a mathematical model of conjugate heat exchange at the turbulent movement of natural gas in a well and the estimation of the intensity of the variation of the performance parameters of the travelling gas.

\section{Problem statement}

Figure 1 shows the design diagram of the production section of the gas well. The section is presented in the form of a vertical tube with the length $L$, inner radius $r_{l}$ and outer radius $r_{2}$. Let us assume that the external temperature $T_{w}$ and the conditions of the heat exchange given via the heat-exchange coefficient $\alpha_{w}$ are known. Through the metal wall of the well, there is heat exchange between the hot gas flow and the ambient; water is taken as the ambient. For the gas flow, the heat-transfer properties, initial values of pressure $p_{l}$, temperature $T_{l}$ and flow rate $\rho u_{l}$ are specified. 


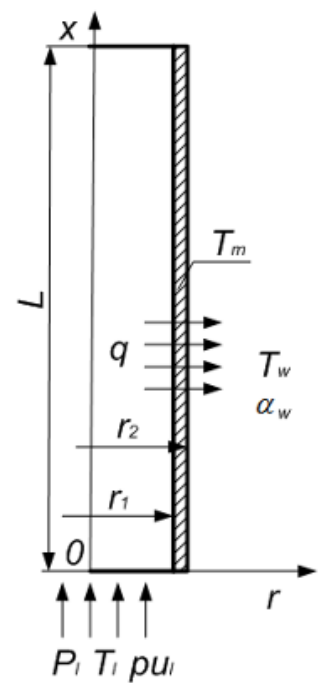

Fig. 1. Design diagram of the production section of the well

\section{Mathematical model}

The mathematical model of the gas flow movement in the two-dimensional axisymmetric formulation based on the boundary-layer equations has the following form [10]:

momentum equation:

$$
\rho u \frac{\partial u}{\partial x}+\rho v \frac{\partial u}{\partial r}=-\frac{d p}{d x}+\frac{1}{r} \frac{\partial}{\partial r} r \rho\left(v_{t}+v\right) \frac{\partial u}{\partial r}-g \rho
$$

continuity equation:

$$
\frac{\partial(\rho u r)}{\partial x}+\frac{\partial(\rho v r)}{\partial r}=0
$$

energy equation:

$$
\rho u \frac{\partial T}{\partial x}+\rho v \frac{\partial T}{\partial r}=\frac{1}{r} \frac{\partial}{\partial r} r \rho\left(\frac{v_{t}}{P r_{t}}+\frac{v}{P r}\right) \frac{\partial T}{\partial r}
$$

The connection between the parameters of the gas flow state is taken as that for the ideal gas:

$$
p=\rho R T
$$

The turbulence one-parameter model $[6,11]$ is written using kinematic turbulent viscosity $v_{t}$.

$$
\rho u \frac{\partial v_{t}}{\partial x}+\rho v \frac{\partial v_{t}}{\partial r}=\frac{1}{r} \frac{\partial}{\partial r} r \rho\left(\chi v_{t}+v\right) \frac{\partial v_{t}}{\partial r}+\alpha \rho v_{t}\left|\frac{\partial u}{\partial r}\right|-\gamma \rho \frac{v_{t}\left(\beta v_{t}+v\right)}{r^{2}}+\xi v_{t} u\left(\frac{\partial \rho}{\partial x}\right)
$$

Here, $\chi, \alpha, \gamma, \beta, \xi$ are empirical coefficients of the turbulence model. The initial and boundary conditions are taken as those in $[10,10]$. The system of equations $(1)-(5)$ is solved using the finite-difference method [12]. The velocity and pressure fields are calculated by L.M. Simuni algorithm [13]. The values of the coefficient $\alpha_{w}$ of the heat 
exchange between the outer wall of the well and the ambient, which is assumed to be water, is determined as in [14].

\section{Results of the numerical calculations}

The section of the vertical well with inner radius $r_{1}=80 \mathrm{~mm}$ and outer radius $r_{2}=100 \mathrm{~mm}$ are taken as initial data. The temperature of the ambient is determined by the natural convection of water relative to the vertical surface of the considered section. The material of the tube is alloy steel. The heat-transfer parameters of the travelling gas are taken as for methane. The initial pressure $p_{l}=100 \mathrm{MPa}$, the temperature $T_{l}=80, T_{w}=0^{\circ} \mathrm{C}$. The calculations are made for the sections of the vertical well with $L: 1-10 \mathrm{~m}, 2-50 \mathrm{~m}, 3-$ $100 \mathrm{~m}, 4-200 \mathrm{~m}$. The initial velocity of the fluid flow is $10 \mathrm{~m} / \mathrm{s}$. Figure 2 shows the temperature distribution along the well radius.

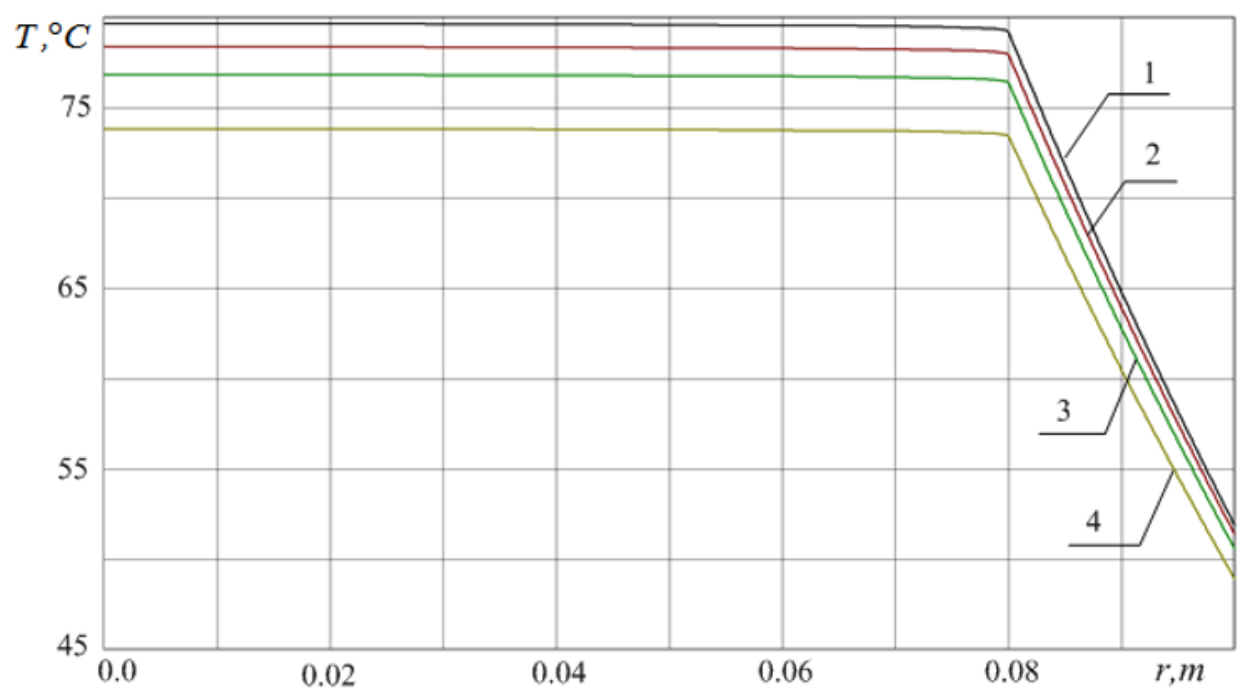

Fig. 2. Profile of the temperature distribution along the radius of mating media

It can be seen that when the gas flow moves upwards in the well the temperature decreases from the initial value. The temperature of the gas flow and inner surface of the well wall remains almost unchanged on the first section. When the gas flow passes $50 \mathrm{~m}$ the temperature drops by almost three degrees. On the section $100 \mathrm{~m}$ the temperature drops by four degrees. On the section $200 \mathrm{~m}$ from the beginning of the movement, the gas temperature decreases by seven degrees at the inner surface of the well wall. Thus, it can be seen that the average gradient of the temperature decrease is about $3.5^{\circ} \mathrm{C} / 100 \mathrm{~m}$. The obtained temperature distribution is mainly determined by the conditions of the external heat exchange.

Figure 3 presents the value of the heat exchange coefficient $\alpha_{w}$ between the outer surface of the well wall and the ambient along the length of the calculation tube section. 


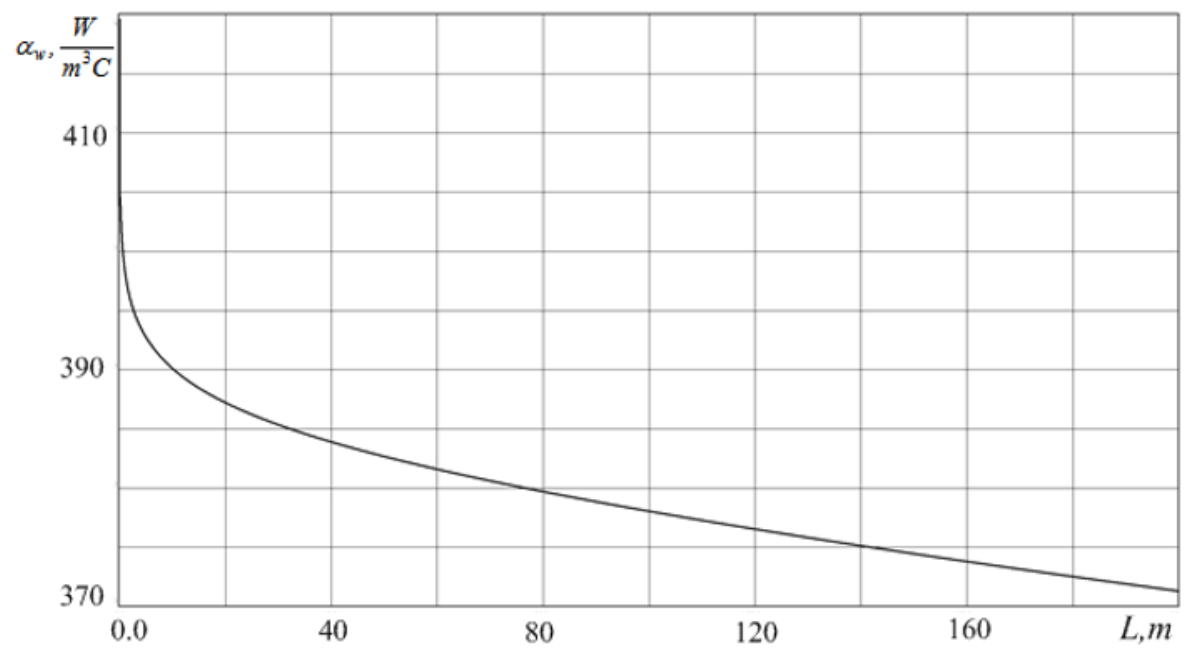

Fig. 3. The coefficient $\alpha_{w}$ value of the heat exchange between the outer surface of the well wall and the ambient along the length of the calculation section

Figure 4 shows the distribution of the gas flow density along the radius of the well for the calculation sections.

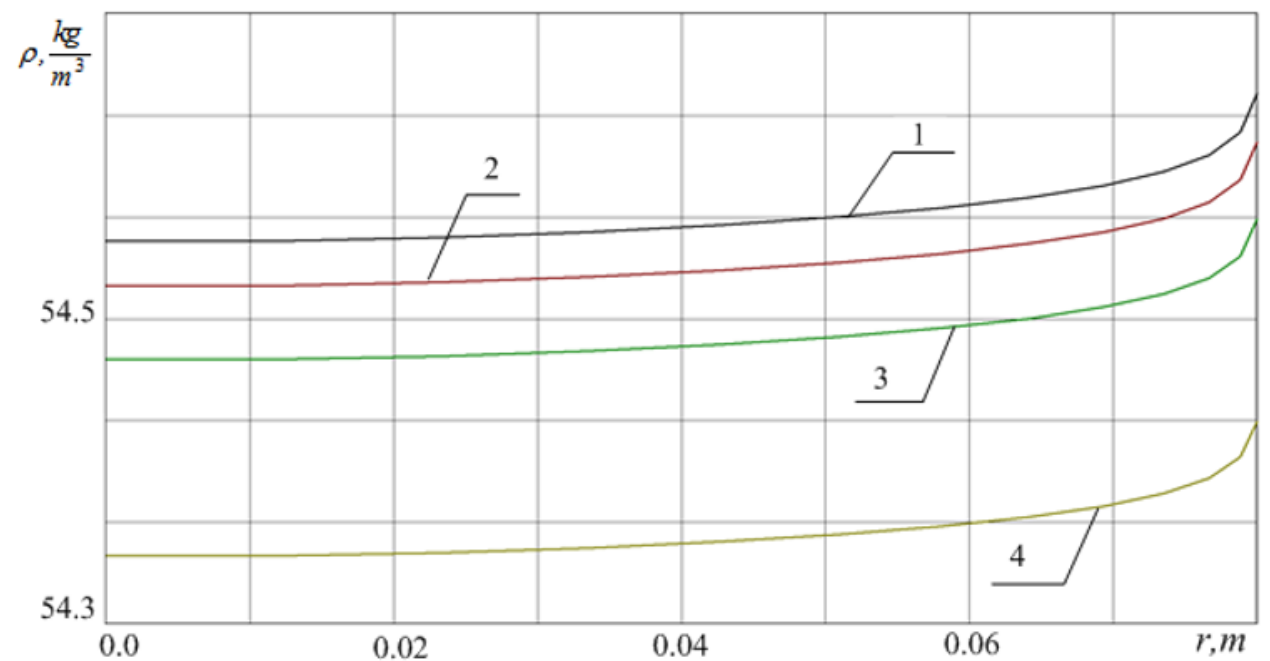

Fig. 4. The distribution of the gas flow density along the well radius

Thus, as the gas flow is moving upwards, the density value is determined by the current values of the pressure and temperature. The obtained density distribution is explained by that the flow density decreases faster than the temperature. Consequently, as can be seen from the obtained results, the gas density value decreases at the upward movement of the gas flow. Due to the temperature influence, the gas density has a larger value at the inner surface of the well wall. In general, on the calculation section the density value decrease is small. 


\section{Conclusion}

In the present paper a mathematical model of the conjugate heat exchange is offered for the natural gas flow moving in the well. The obtained results describe thermobaric state of the well in the condition of the conjugate heat exchange between the gas flow and the wall of the vertical well. The presented model permits to analyze the intensity of the variation of the thermophysical parameters depending on the channel geometry and thermophysical properties of the substances, to determine the distribution of the velocity, temperature and density of the gas flow at the specified parameters of operation.

This work was supported by the Russian Foundation for Basic Research, project No. 20-08 00481a

\section{References}

1. Filippov A.I., Akhmetova O.V., Zelenova M.A., Krupinov A.G. Oil and Gas Business $6(2011)$

2. Filippov A.I., Akhmetova O.V., Olefirenko K.V. Scientific and Technical Volga region Bulletin 4 (2014)

3. Ponomarev A.I., Zaripova K.R. Oil and Gas Business 3 (2013)

4. Fayzullin N. F., Ramazanov A. Sh. Vestnik Bashkirskogo universiteta. 22 (2017)

5. Salenko S.D., Gosteev Y.A., Pavlyuchik M.S. Proceedings of Higher Educational Institutions. Machine Building 1 (2014)

6. Victor W. Nee, and Leslie S. G. Kovasznay The Physics of Fluids 12, 473 (1969)

7. Lumley J.L., Khajeh-Nouri B. Computational modeling of turbulent transport (1974)

8. Bradshaw P., Cebeci T., Whitelaw J.H. Engineering calculation methods for turbulent flow (1981) 9. Wilcox D. C. Turbulence Modeling for CFD (1998)

9. Alies M. Y., Makarov S.S., Karpov A.I Journal of Physics: Conference Series 1679 052028 (2020)

10. Alies M.Y., Makarov S.S., Karpov A.I. MATEC Web of Conferences, 329, 03013 (2020)

11. Abramovich G. N., Krasheninnikov S. Yu., Secundov A. N. Turbulent flows under the influence of volumetric forces and nonautomodelnost (1975)

12. Samarsky A. A., Vabishchevich P. N. Computational heat transfer (2003)

13. Simuni L. M. USSR Computational Mathematics and Mathematical Physics 5 (1965)

14. Mikheev M. A., Mikheeva I. M. Fundamentals of heat transfer (1977) 